\title{
CELLULAR AUTOMATA: RETINAL CELLS, CIRCULATION AND PATTERNS
}

\author{
MARTIN A. MAINSTER \\ Kansas City, Kansas, USA
}

\begin{abstract}
SUMMARY
Cellular automata modelling is a useful mathematical technique for simulating complex biological systems. An area to be studied is broken into a lattice of adjacent cells depicted by picture elements on a computer screen. The initial tissue pattern evolves on the computer screen, directed by a rule that considers the state of each cell and its neighbours in the lattice. Simulations of wound repair, cell proliferation, retinal circulation and pigment aggregation serve to illustrate the potential value of cellular automata modelling in ophthalmic research and practice.
\end{abstract}

Mathematical simulation can provide insights into disease and therapy, but most physicians have a healthy scepticism for mathematical models of medical phenomena. Models are typically cloaked in esoteric terms and methods, seldom having real world clinical value. Realistic medical models are difficult to formulate for many reasons, but the complexity of biological systems is the greatest obstacle. In the past decade, new methods have been developed for describing complex systems, ${ }^{1-6}$ and there is new hope for devising computer models that are useful in the complex world of clinical practice.

Mathematical models usually have a top-down or bottom-up perspective. ${ }^{7-9}$ In top-down, phenomenological methods, a system is studied experimentally, inferences made about observations, and a model relating the system's variables devised to predict the system's behaviour. Examples of this empirical approach include multiple regression models for predicting intraocular lens power $^{10}$ and neural networks or expert systems for diagnosing retinal disease. ${ }^{11}$ In bottom-up, ontological methods, key variables and their relationships are postulated, system behaviour calculated, and model performance improved by comparing predictions with experimental observations. Examples of this theoretical approach are heat conduction models used to calculate chorioretinal $^{12,13}$ or corneal ${ }^{14}$ temperature increases from laser exposure.

Correspondence to: Martin A. Mainster, M.D., Ph.D., Department of Ophthalmology, Kansas University Medical Center, 39th and Rainbow Boulevard, Kansas City, Kansas 66103, USA.
In the physical sciences, mathematical models serve as vehicles for testing theories and designing experiments. Mathematical insights often anticipate experimental progress. For example, quantum mechanics was the foundation for the discovery of lasers and semiconductors. Traditional bottom-up, reductionistic reasoning assumes that systems can be understood fully by studying their individual parts independently. This approach fails, however, when confronted by complex phenomena such as weather systems or turbulent blood flow in retinal vessels.

A biological system can be thought of as a dynamical system. A dynamical system has a state and a dynamic. ${ }^{1}$ Its state is specified by the values of variables needed to describe the system at a particular time. Its dynamic is a set of equations or rules that determine how the variables change with time. The variables that specify the system's state define a multidimensional phase (or state) space. Thus, the evolution of a dynamical system may be viewed as a trajectory (or orbit, or path) in phase space. For example, the clinical history of an individual retina may be represented as a trajectory in retinal phase space, altered by growth, ageing, disease and therapy. The development of effective mathematical models of retinal behaviour requires identifying the key structural and physiological variables that define retinal phase space.

Mathematical models can be classified as continuous or discrete. The dynamic of a continuous model is usually a set of differential equations that governs how the system's state evolves continously in space and time. Continuous models provide valuable descriptions of heat flow, tissue deformation and other phenomena describable by differential equations. ${ }^{8,14}$ The dynamic of a discrete model is usually a set of difference equations ${ }^{6,8,12}$ (a mapping) or a cellular automaton ${ }^{5-7,15-18}$ that governs how the system evolves in individual time steps when space is divided into individual cells.

Mathematical models can also be classified as deterministic or indeterministic. ${ }^{8}$ In deterministic models, variables are governed by equations that completely specify their future values. In indeterministic (or stochastic) mod- 
els, randomness is built into the relationships of the variables and their temporal evolution. A great advance in the past two decades has been recognition that completely deterministic systems can be quite unpredictable. ${ }^{1-4}$ Inaccuracies are introduced by the limited precision with which a system's variables can be specified at any one time. This imprecision eventually dominates the system's behaviour, a phenomenon known as deterministic chaos. Since chaotic systems can appear to have random behaviour, seemingly random patterns in clinical systems may arise from simple forces governing complex systems, and these patterns may hold the key to understanding underlying forces and devising therapeutic strategies.

Cellular automata simulations are discrete, deterministic mathematical models, powerful new alternatives to differential equations. Automata can solve the same types of problems as differential equations, but they may be better suited to simulating biological systems with many degrees of freedom on contemporary serial and emerging parallel computer architectures. Cellular automata have been used to simulate hydrodynamics and thermodynamics, $7,15-17$ chemical reactions, ${ }^{19}$ autoimmunity,${ }^{20}$ tumor growth ${ }^{21}$ and excitable neural media. ${ }^{22}$ In this report, the usefulness of cellular automaton modelling in ophthalmic research and practice will be demonstrated in simulations of tissue repair, cell proliferation, retinal blood flow and pigment aggregation.

\section{METHODS}

\section{Automata basics}

In a cellular automaton simulation, a region to be studied is broken up into a uniform lattice of adjacent cells. In two dimensional automata, each cell in the lattice may be represented by a single pixel (picture element) on a computer screen. Each cell can occupy one of a finite number of states, depicted by the colour of the pixel representing it. An initial screen pattern is formed by assigning a state to each cell. The initial screen pattern is known as the first, generation of a cellular automaton simulation.

The initial screen pattern (first generation) of an automaton is changed to a second screen pattern (second generation) according to a rule. This change represents a single time step in the automaton's evolution. The rule selects the state of each cell in the second generation according to the state of the cell and its eight neighbouring cells in the first generation. The third generation is determined by applying a rule to the second generation, and so on. Thus, a cellular automaton pattern evolves in discrete time steps, generation by generation, when a rule is simultaneously, independently and uniformly applied to each lattice cell. ${ }^{67,15}$ The appearance of the pattern changes (grows, evolves) on the computer screen as the state of cells changes from one generation to the next. Different rules produce different types of changes in cell patterns.

\section{Automata rules}

Cellular automata use totalistic, semitotalistic, and par- titioning rules. ${ }^{7,15,23}$ Totalistic rules treat each cell of the lattice equally. When a calculation is performed to determine a cell's state in the next generation, the computer accesses the same amount of information in the current generation about that cell and each of its eight neighbours. Memory requirements increase rapidly when more than one bit of data are used for each cell in the lattice. Semitotalistic and partitioning cellular automata rules are ways to increase the complexity of simulations with the limited memory available in contemporary microcomputers.

In a semitotalistic automaton, the computer accesses more information about the cell to be updated than its neighbours. In this report, an automaton will be used with eight bits of information about a cell and one bit of information about each of the cell's neighbours (a total of 16 bits). This limited data on neighbouring cells still restrict simulations to very simple systems.

In a partitioning cellular automaton, the lattice is divided into blocks of adjacent cells, and the algorithm operates on blocks rather than individual cells. ${ }^{7,15,16,24,25}$ Lattice partitioning is used in lattice gas models that simulate particle movement on the computer screen. Lattice gases provide an alternative to differential equations models of certain systems. ${ }^{15,16}$ In the simulations presented in this report, the lattice is divided into blocks of four cells, and particles move, bounce off each other, and rebound from walls (boundaries made up of pre-selected cells) according to a purely deterministic automata algorithm, the HPP-gas model. ${ }^{7,15,24}$

In the HPP model, particle number and momentum are unchanged (conserved) in collisions. Initial particle distribution may be uniform or random. HPP particles obey the Navier-Stokes partial differential equation for the flow of a viscous fluid, ${ }^{7,15,24}$ but the simulation is only approximate for the square lattice used in this report. An exact simulation requires a more complicated hexagonal lattice model to assure that viscosity is isotropic..$^{7,15,25}$

\section{Software details}

Cellular automata simulations were carried out on a $320 \times$ 200 pixel lattice $(64,000$ cells), using the Autodesk CA Lab program ${ }^{7}$ on a Zenith $8038625 \mathrm{MHz}$ microcomputer with VGA graphics. Computation speed varies, but typically is five generations/second. Eight bits of data are available for each cell (a bit is a 1 or a 0 ), arranged into eight bit planes as shown in Figure 1. Each of the eight bits of data can represent a different cell characteristic.

The combined binary data in each bit plane for a particular cell determines the state of that cell, a number between zero and 255 . The computer screen pattern is formed by assigning a colour to the pixel representing each cell in the lattice, using a drawing utility and a palette that specifies a particular colour for each of the states that the cell can occupy. The palette can be changed to emphasise or suppress information in a particular bit plane. Since the eight bit planes are independent, the screen pattern actually represents the eight separate patterns in each bit plane. Eight bit plane buffers are available for storing, pro- 


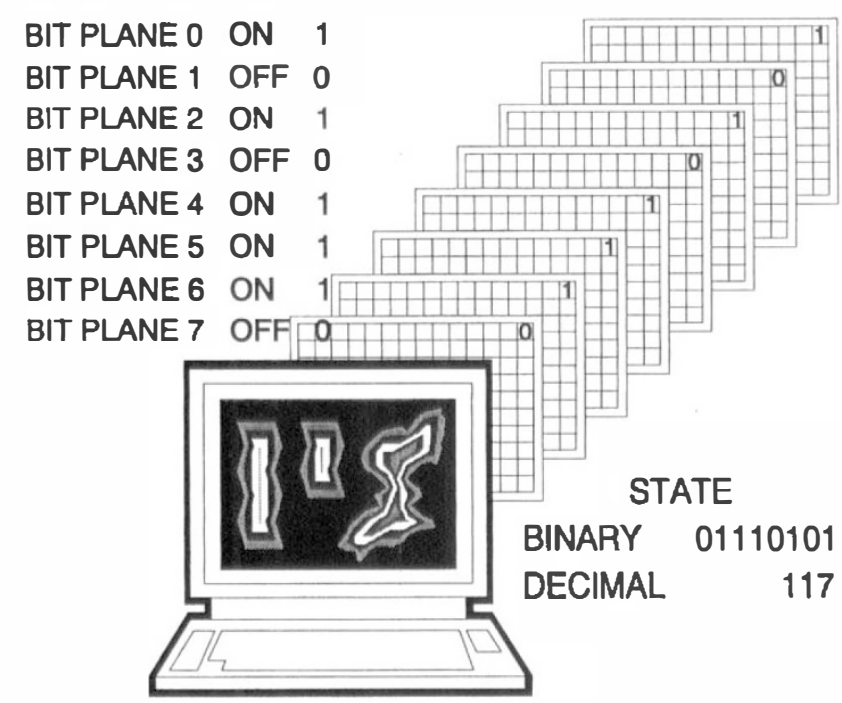

Fig. 1. Cellular automata can be used to simulate clinical problems. An area to be simulated is broken up into a lattice of adjacent cells. Each picture element on the computer screen depicts the state of one cell. An initial screen pattern shows the state of each cell in the lattice. The initial pattern evolves in a series of time steps according to a rule that considers the state of each cell and its 8 neighbours. In the CA Lab program, a maximum of 256 states are available for each cell, providing 8 independent bit planes for storing different types of information about each cell.

cessing or displaying patterns. Rules, patterns and palettes can be stored, recalled or interchanged independently at any point in a simulation. Cellular automata rules were written in the Pascal language.

Two dimensional cellular automata geometries were used in this report, although meaningful biological patterns may be obtained with one and three dimensional automata. ${ }^{21,26}$ A closed (toroidal) lattice topology was used, but identical simulations can be performed with an open (non-toroidal) screen topology. With a toroidal lattice, the top edge of the lattice (screen) wraps around and connects to its bottom edge, and the right edge of the lattice connects to its left edge. With a non-toroidal geometry, the lattice ends at the edge of the screen.

Computer screen patterns were printed on a HewlettPackard LaserJet II with an Adobe PostScript cartridge. Patterns were transferred to the printer as postscript files or converted to TIFF bitmaps with Applications Technology Pizazz Plus for editing with Micrografx Designer and subsequent PostScript conversion.

\section{RESULTS}

Figure 2a presents a tissue repair simulation performed with a simple, one bit, totalistic algorithm. The automaton obeys a simple type of counting (voting) rule. ${ }^{27}$ If a cell is occupied, it remains so; if it is unoccupied, it becomes occupied if two or more neighbouring cells are occupied, or else it remains unoccupied. One bit of data is available to describe each cell and its eight neighbours (a total of nine bits). This automaton demonstrates two types of behaviour seen in cell layers: (1) if a hole is present in a field of occupied cells, the field of occupied cells spreads inward to repair the defect; and (2) if one or more fields of cells are present in an unoccupied region, the fields expand to fill the region.

The outer border of each of the three patterns in Figure $2 a$ is the original 'wound' size. The outer border of each of the more lightly shaded regions is the size of the wound after 10, 20 and 30 generations of cell growth (40 and 50 generations are also shown for the larger lesion). Figure 2a shows that automata can accommodate initial patterns of arbitrary complexity. For the two wounds on the left side,

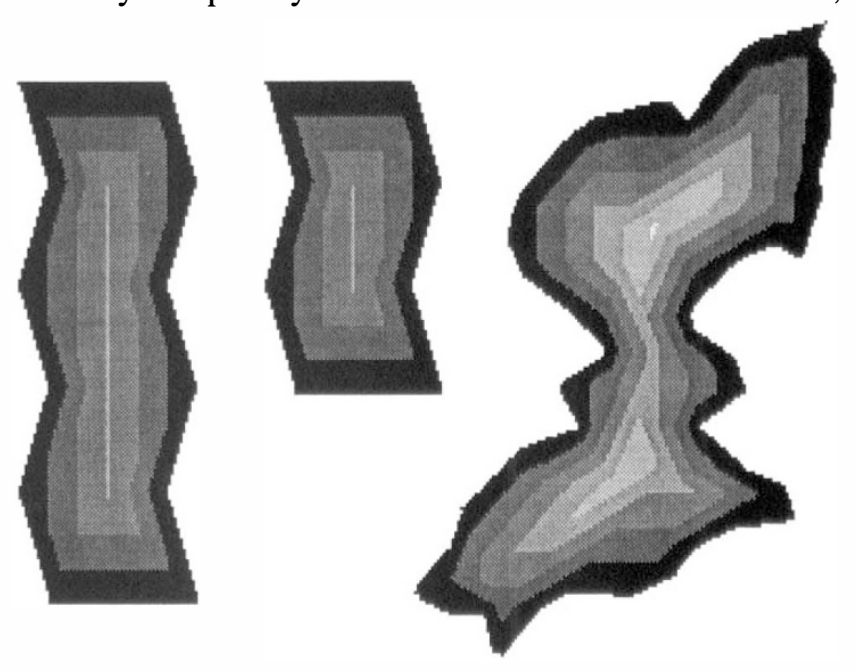

Fig. $2 a$.

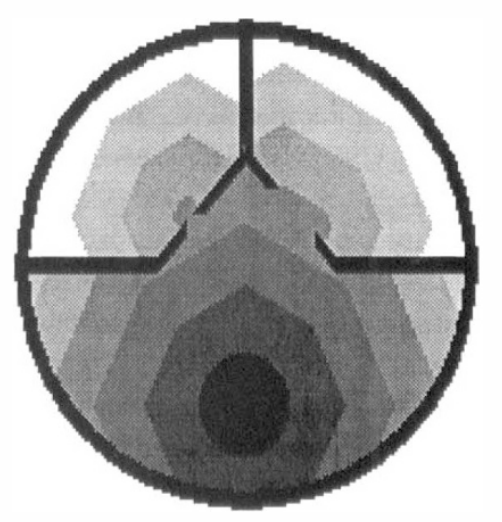

Fig. $2 b$.

Fig. 2. Cellular automata simulation of tissue repair and cell culture growth. In Fig. 2a, the outer border of each of the three patterns is the original size of an unoccupied region or 'wound' in an otherwise intact tissue layer. The outer border of each of the more lightly shaded regions is the size of the wound after 10, 20 , and 30 generations of cell growth $(40$ and 50 generations are also shown for the larger lesion). Automata can accommodate initial patterns of arbitrary shape. The two lesions on the left side demonstrate that the duration of wound repair depends on the width not the length of a linear incision. In Fig. 2b, a field of cells (the black ellipse) expands within a circular dish that has interrupted linear partitions. The shaded regions show the size of the expanding tissue layer after 35, 70, 105, 140 and 175 generations. 


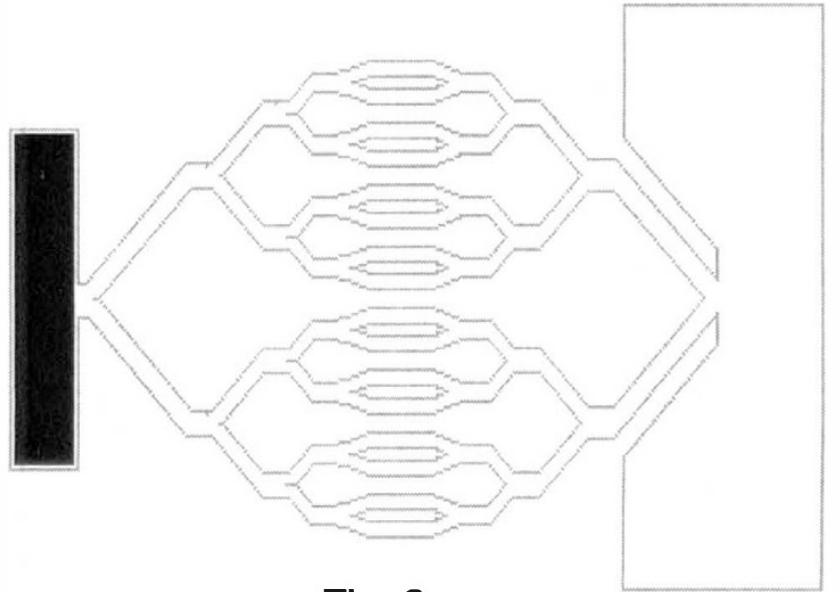

Fig. 3a.

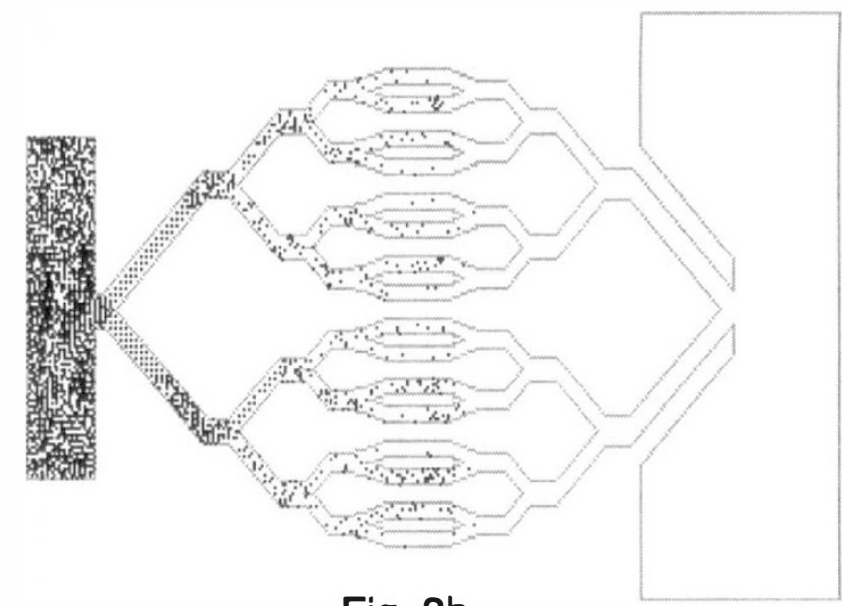

Fig. 3b.

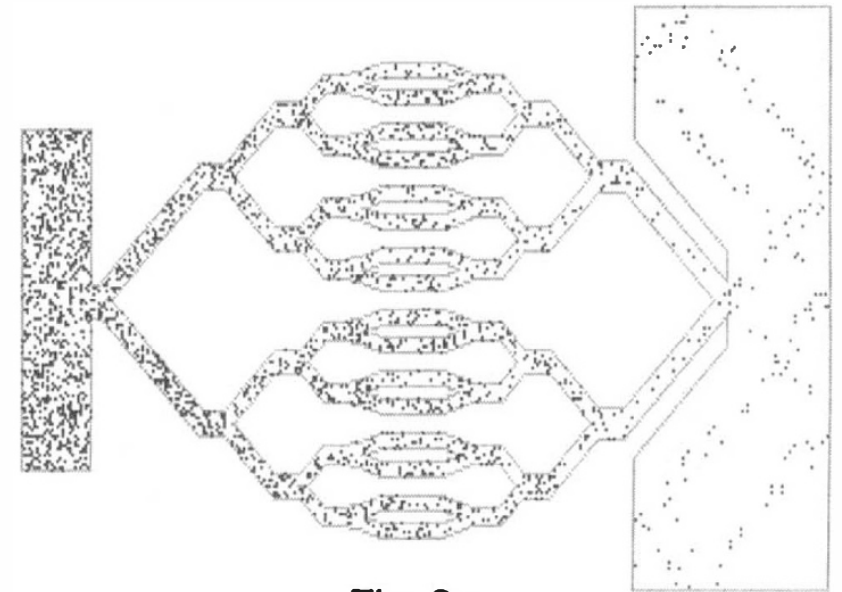

Fig. 3c.

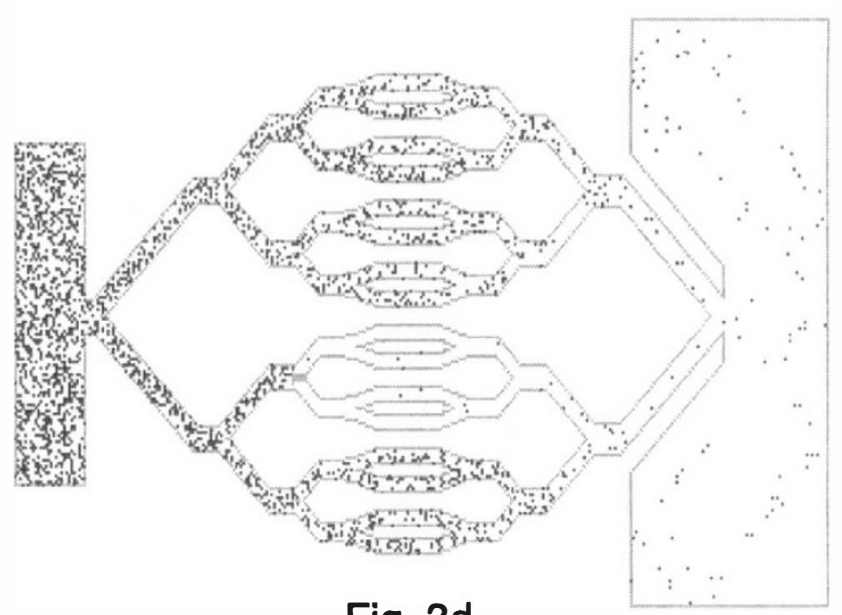

Fig. 3d.

Fig. 3. A simple lattice gas simulation of retinal circulation. In Fig. 3a, the black rectangle at the left side is an initial collection of 2568 particles representing fluorescein in a central retinal artery. Fig. 3b shows the arterial phase (450 generations) and $\mathbf{F i g} .3 \mathbf{c}$ shows late transit phase (1450 generations) of particle flow. Fig. 3d is the late transit phase (1450 generations) of a simulated inferior branch retinal artery occlusion, showing reduced arterial flow and retrograde venous filling.

Figure 2 also shows that the duration of wound healing depends on the width rather than the length of a linear incision.

Figure $2 b$ presents a semitotalistic variation of the automaton in Figure 2a. Eight bits of data are used for each cell and one bit for its eight neighbours (a total of 16 bits). The extra seven bits of information provide fixed boundaries for the totalistic algorithm used in Figure 1a. In Figure 2b, a field of cells (the black ellipse) expands within a circular dish that has interrupted linear partitions. The shaded regions show the size of the expanding tissue layer after 35, 70, 105, 140 and 175 generations.

Figures 3 and 4 are HPP lattice gas simulations ${ }^{7,15,24}$ of vascular networks in the retina, using the HPP model described above. The black rectangle at the left side of Figure $3 \mathrm{a}$ is an initial collection of 2568 particles used to simulate fluorescein in a central retinal artery. Figures $3 \mathrm{~b}$ and $3 \mathrm{c}$ show arterial (450 generations) and later transit phases (1450 generations) of particle flow. Flow from left to right through the vascular network is driven by the higher particle density on the arterial than the venous side. Figure $3 d$ is the late transit phase (1450 generations) of a simulated inferior branch retinal artery occlusion, illustrating decreased local arterial flow and retrograde venous filling.

Figure 4 is a simple simulation of foveal circulation in which four blocks of particles (1512 particles/block) enter a rotary capillary network through four radial arterioles and exit through four radial venules. Figures $4 a$ and $4 b$ show arterial (250 generations) and late transit phases (1250 generations) of the simulation. Figure $4 \mathrm{c}$ is the late transit phase of a simulation (1250 generations) where there is a leaking microaneurysm inferiorly. Particles that leak from the microaneurysm accumulate in adjacent parafoveal space, a simulation of parafoveal telangiectasis with oedema. Figure 4d shows the late transit phase (1250 generations) of a similar computation after partial closure of the microaneurysm (as in laser therapy). Fewer particles accumulate in parafoveal space when the channels on either side of the leaky microaneurysm are constricted.

Figure 5 was computed from an HPP lattice gas model modified to simulate aggregation. ${ }^{7}$ Particles still collide and bounce off each other, but now they stick to preselected aggregation walls or individual cells when they 


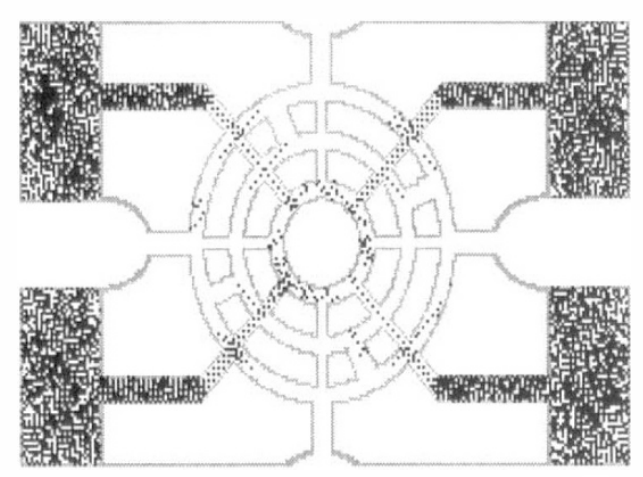

Fig. 4a.

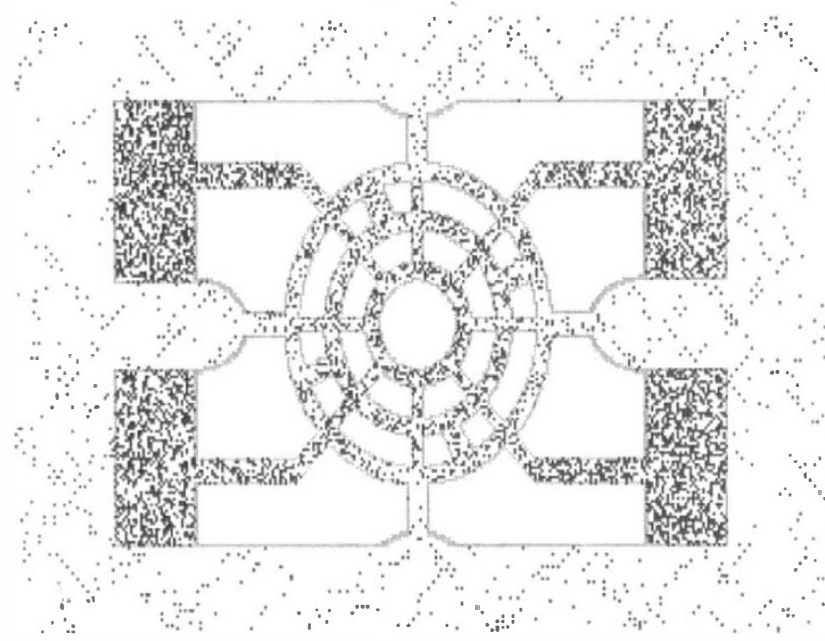

Fig. 4b.
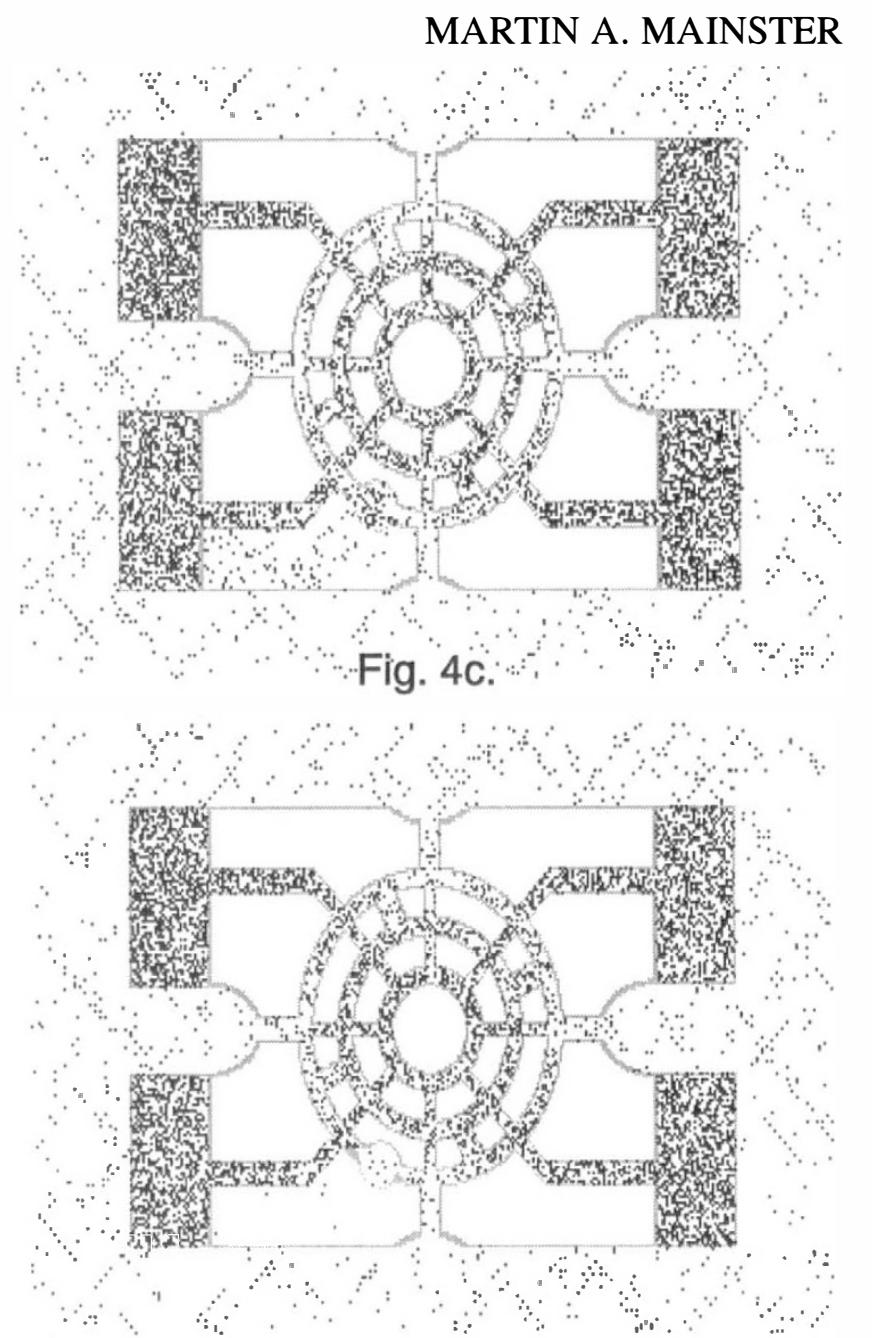

Fig. 4d.

Fig. 4. A simple lattice gas simulation of foveal circulation. Particles representing fluorescein enter a rotary capillary network through 4 radial arterioles and exit through 4 radial venules. Fig. $4 \mathbf{a}$ shows the arterial phase (250 generations) and $\mathbf{F i g} .4 \mathbf{b}$ shows the late transit phase (1250 generations) of the simulation. Fig. $4 \mathbf{c}$ is the late transit phase (1250 generations) of the system modified by the presence of a leaky microaneurysm inferiorly, resulting in parafoveal fluorescein accumulation. Fig. 4d shows the late transit phase (1250 generations) of a similar simulation after partial closure of the microaneurysm. Less fluorescein accumulates in parafoveal space after channels on either side of the leaky microaneurysm are constricted as in laser therapy.

encounter them. Thus, particles move across the screen' until they strike and become part of the walls or cells, producing aggregation patterns that grow in size.

Figure $5 \mathrm{a}$ is the initial pattern for an HPP-gas aggregation automaton, showing a vascular pattern (walls), a ring of aggregation points temporal to the macula (point walls), a collection of particles within the aggregation ring, and a random layer of particles that increases in density from the centre to the periphery of the figure. Figure $5 \mathrm{~b}$ shows the automaton after 40 generations of growth, illustrating aggregated particles along vessel walls and in a circinate pattern around the aggregation points. Perivascular pigment clumpng from lateral pigment migration is seen in retinitis pigmentosa and pigmented paravenous atrophy. Circinate rings may occur in diabetic retinopathy when water removal at the periphery of an area of oedema leaves a ring of lipid residue at the perimeter of the oedematous region.

Lattice gas models can also be used for diffusion limited aggregation (DLA) computations. ${ }^{15}$ In DLA, a single aggregation cell is placed in the centre of the lattice, and a particle does a random walk from a random location on a circle centered about the cell. If the particle strikes the circle, it bounces back toward the centre of the circle. When the particle strikes the aggregation cell, it stops and becomes part of a two cell aggregation pattern. Additional particles are released one at a time from random locations around the circle, attaching to a growing structure. ${ }^{28-31}$ The circle is increased in diameter as the pattern grows in lateral extent.

The pattern in Figure 6 is an enlarging diffusion limited aggregation pattern, ${ }^{28-31}$ computed directly from the DLA algorithm without a partitioning automaton. ${ }^{32}$ DLA patterns have a fractal dimension (D) of $1.68 \pm 0.05,{ }^{33}$ spanning 2-dimensional space more effectively than a line $(\mathrm{D}=$ 1) but less effectively than a disk $(D=2)$. In Figure 6 , a DLA pattern and its fractal dimension are shown at three different growth stages. DLA-like patterns occur in a variety of situations governed by Laplace's equation such as dielectric breakdown ${ }^{34}$ and viscous fingering. ${ }^{35}$ Retinal 


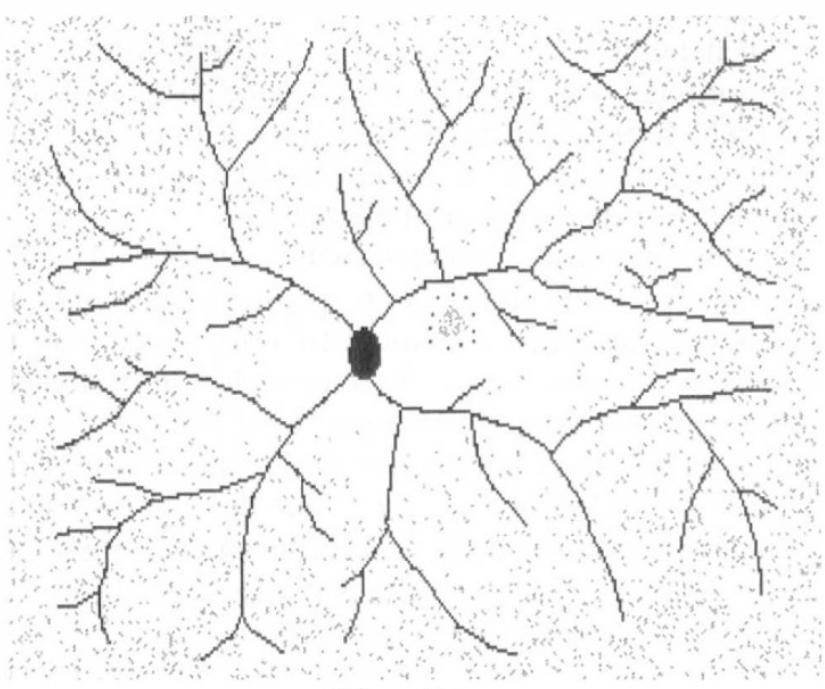

Fig. 5a.

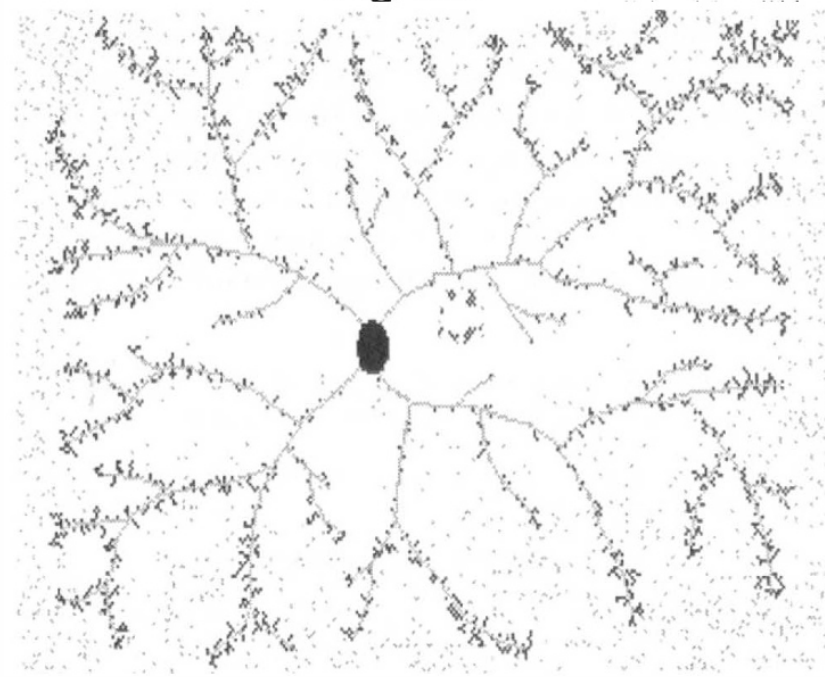

Fig. 5b.

Fig. 5. A lattice gas simulation of retinal pattern formation. Fig. 5a shows an initial vascular pattern, an initial ring of aggregation points temporal to the macula, a collection of particles within the aggregation ring, and a random layer of particles that increases in density from the centre to the periphery of the figure. Fig. 5b shows the automaton after 40 generations of growth, illustrating aggregation of particles along vessel walls as in pigmented paravenous atrophy and in circinate pattern around aggregation points as in diabetic retinopathy.

arterial, venous and capillary patterns have fractal dimensions of $1.63,1.71$, and 1.82 , respectively, suggesting that arteriole and venule formation are Laplacian processes but other factors predominate in capillary growth. ${ }^{31}$

\section{DISCUSSION}

The objective of mathematical simulation is accurate prediction of a biological system's future behaviour. From a practical perspective, simulations are useful only when they are much simpler and faster than original systems. Clinical simulations have numerous potential applications. For example, a retinal simulation could be used to test the efficacy of different laser patterns for treating parafoveal telangiectasis or diabetic macular oedema. In the past, biological systems have been far too complex for conventional differential equation modelling. This outlook has changed with new mathematical techniques and powerful interactive computers.

Cellular automata are discrete, deterministic, local mathematical models. Every pixel on the computer screen represents the state of a cell in the automaton lattice. An automaton is discrete in three ways: (1) each cell occupies one of a finite number of states, (2) space is broken into individual cells, and (3) an initial pattern evolves in individual time steps (generations) when the same rule is uniformly and simultaneously applied to each lattice cell.

Cellular automata models may be computationally irreducible $^{36,37}$ (unsimulatably complex) ' $^{9}$ that is, there may be no way of computing an automaton's future pattern that is faster than letting it evolve. For certain systems, it may be possible to develop reversible automata that run backwards in time to identify the patterns that lead to pathol-
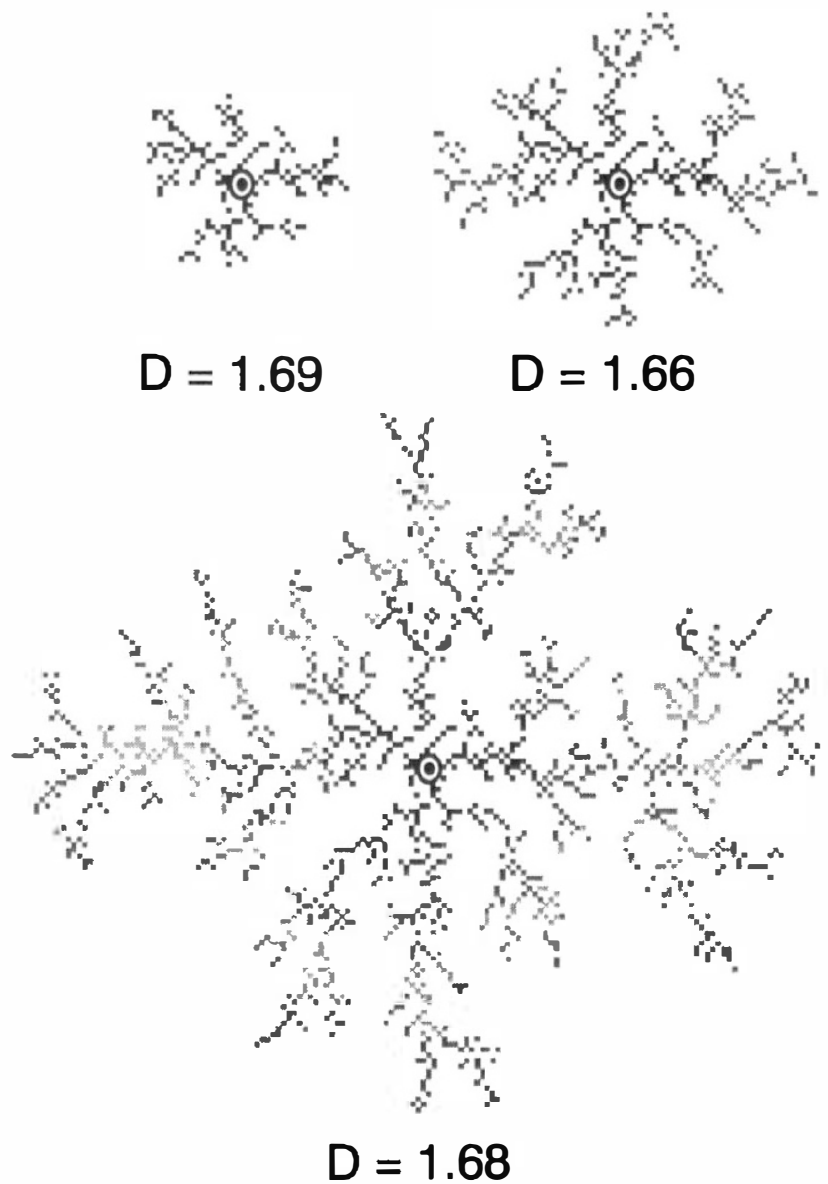

Fig. 6. An enlarging diffusion limited aggregation (DLA) pattern, formed when particles stick one by one to a single aggregation cell in the center of a lattice. Large DLA patterns have a fractal dimension $(D)$ of $1.68 \pm 0.05$, spanning 2-dimensional space more effectively than a line $(D=1)$ but less effectively than a disk $(D=2)$. DLA growth patterns occur in nature when processes are governed by Laplace's equation. Retinal arterial, venous and capillary patterns have fractal dimensions of $1.63,1.71$, and 1.82 , respectively, suggesting that arteriole and venule formation are Laplacian processes but that other factors predominate in capillary growth. 
ogy. Much of the recent interest in cellular automata is due to the success of lattice gas models in describing complex physical processes such as fluid flow in three dimensional and multiple-fluid systems. ${ }^{16,17}$

In a certain sense, cellular automata simulation is image processing $^{38}$. Image processing involves digitising a picture (breaking it into a finite number of pixels) and changing pixel darkness (or colour) to emphasise or suppress certain types of information. Cellular automata also operate on images, the initial screen patterns. An automaton uses a local rule (based on the state of each cell and its neighbours) to transform the initial screen pattern into the screen patterns of subsequent generations.

The ability to change an automaton rule (a dynamic) or modify a pattern (the system's state) between generations provides remarkable flexibility for clinical simulation. Since automaton rules are local, an automaton may be thought of a special case of a neural network, where nonlocal interactions are permitted. ${ }^{6,11}$ Far more complex systems could be simulated by generalising automata to permit non-uniformity and non-locality. Microscopic detail could be simulated in one grid region without excessive cell numbers by exponentially stretching the lattice away from the region. ${ }^{12}$ Non-ocular effects could be accommodated by permitting an automaton rule to access information from distant lattice locations. The distant locations could represent systemic effects evolving independently with different algorithms in isolated lattice partitions.

Ophthalmic practice involves identifying abnormal tissue patterns and treating them when indicated. Recognising and classifying biomicroscopic and ophthalmoscopic patterns remain largely subjective processes in contemporary practice, but digital imaging offers a foundation for more objective analyses. A digitised image can be quantified by using a computer to count structures such as microaneurysms, ${ }^{39}$ to measure areas such as the lateral extent of macular exudates ${ }^{40}$ or non-perfusion, ${ }^{41}$ to assess the spatial frequency of repetitive structures such as the retinal nerve fibre layer, ${ }^{42}$ and to determine the fractal dimension of space filling patterns such as the retinal arterial and venous arcades. ${ }^{31}$

Image analysis is a valuable phenomenological source of data for theoretical modelling. Cellular automata are well suited to using this data to test theories of pathogenesis and therapy. It might be argued that the automata in Figures 2-5 are more caricature than mathematical model. Tissue proliferation certainly depends on many factors other than the presence of adjacent cells, and capillary circulation is far more complex than the flow of a viscous fluid through a rigid tube. Nonetheless, the simulations are of heuristic value, and with few assumptions about structure and interaction, they produce patterns remarkably similar to those seen in clinical practice.

The simplicity of the simulations in Figures $2-5$ is imposed by current microcomputer constraints, not theoretical limitations. For example, a scanning laser ophthalmoscope fluorescein angiogram ${ }^{43-45}$ of a patient with parafoveal telangiectasis could provide the basis for the first generation of a lattice gas simulation. Alternative forms of focal laser therapy could then be tested to determine which provided the desired perturbation of retinal circulation with the least damage to underlying photoreceptors and retinal pigment epithelium.

Cellular automata simulations with sophisticated parallel computer architectures can be performed currently at speeds $10^{4}$ times faster on lattices $10^{6}$ times larger than in this microcomputer study. ${ }^{17}$ With enough states for each lattice cell, automata simulations could address factors such as chemical mediation in wound repair, pulsation in blood flow, turbulence at arteriovenous crossings that might lead to retinal branch vein occlusion, and migration and aggregation of debris in pigmentary or pseudoexfoliation glaucoma or macular degeneration.

A cellular automaton is a metaphor on nature in binary arithmetic, no more or less real than any other rational conceptualisation of reality. Since an automaton may be computationally irreducible, it may also offer the simplest mathematical description obtainable for a system. Automata are vehicles for testing our understanding of reality, important members of an emerging new generation of experimental mathematical techniques that may dramatically expand the role of computer modelling in clinical practice.

This research was supported in part by Kansas Lions Sight Foundation, Inc. and Research to Prevent Blindness, Inc.

Key words: Cellular automata, Chaos, Circulation, Fractal, Lattice gas, Mathematical modelling, Retina.

\section{REFERENCES}

1. Crutchfield J, Farmer JD, Packard NH, Shaw RS: Chaos. Scientific Am 1986 (Dec), 255: 46-57

2. Goldberger AL, Rigney DR, West BJ: Chaos and fractals in human physiology. Scientific Am 1990 (Dec), 262: 42-49.

3. Schroeder M: Fractals, Chaos, Power Laws. New York, WH Freeman, 1991.

4. Briggs J, Peat FD: Turbulent Mirror. New York, Harper and Row, 1989.

5. Wolfram S: Computer software in science and mathematics. Scientific Am 1984 (Sept), 251: 188-203.

6. Rietman E: Exploring the Geometry of Nature-Computer Modelling of Chaos, Fractals, Cellular Automata and Neural Networks. Blue Ridge Summit (PA), Windcrest, 1989.

7. Rucker R, Walker J: CA Lab. Sausalito (CA), Autodesk, 1989.

8. Morrison F: The Art of Modelling Dynamic Systems-Forecasting for Chaos, Randomness and Determinism. New York, Wiley-Interscience, 1991.

9. Pagels HR. The Dreams of Reason-The Computer and the Rise of the Sciences of Complexity. New York, Simon and Schuster, 1988.

10. Retzlaff JA, Sanders DR, Kraff MC: Development of the SRK/T intraocular lens implant power calculation formula. J Catract Refract Surg 1990, 16: 333-40.

11. Goldbaum MH, Katz NP, Nelson MR, Haff LR: The discrimination of similarly coloured objects in computer images of the ocular fundus. Invest Ophthalmol Vis Sci 1990, 31: 617-23.

12. Mainster MA, White TJ, Tips JH, Wilson PW: Transient thermal behaviour in biological systems. Bull Math Biophys 1970, 32: 303-14. 
13. Mainster MA, White TJ, Allen RG: Spectral dependence of retinal damage produced by intense light sources. J Opt Soc Am 1970, 60: 848-55.

14. Mainster MA: Ophthalmic applications of infrared lasersthermal considerations. Invest Ophthalmol Vis Sci 1979, 18: 414-20.

15. Toffoli T, Margolus N: Cellular Automata Machines-A New Environment for Modeling. Cambridge (MA), MIT Press, 1987.

16. Boghosian BM: Lattice gasses. In, Jen E, ed: 1989 Lectures in Complex Systems. Redwood City (CA), Addison-Wesley, 1989: 293-324.

17. Chen H, Chen S, Doolen GD, Matthaeus WH: Lattice-gas models for multiphase flows and magnetohydrodynamics. In, Jen E, ed: 1989 Lectures in Complex Systems. Redwood City (CA), Addison-Wesley, 1989: 389-399.

18. Campbell DK: Nonlinear science-From paradigms to practicalities. In, Cooper NG, ed: From Cardinals to Chaos-Reflections on the Life and Legacy of Stanislaw Ulam. Cambridge, Cambridge University Press, 1989: 218-262.

19. Madore BF, Freedman WL: Computer simulations of the Belousov-Zhabotinsky reaction. Science 1983, 222: 1615-16.

20. Cohen IR, Atlan H: Network regulation of autoimmunity: an automaton model. J Autoimmun 1989, 2: 613-25.

21. Duchting W, Vogelsaenger T: Analysis, forecasting, and control of three-dimensional tumor growth and treatment. $J$ Med Systems 1984, 8: 461-75.

22. Gerhardt M, Schuster H, Tyson JJ: A cellular automaton model of excitable media including curvature and dispersion. Science 1990, 247: 1563-6.

23. Wolfram S: Universality and complexity in cellular automata. Rev Mod Phys 1983, 55: 601-44.

24. Hardy J, de Pazzis O, Pomeau Y: Molecular dynamics of a classical lattice gas: transport properties and time correlation functions. Phys Rev 1976, A13: 1949-60.

25. Frisch U, Hasslacher B, Pomeau Y: Lattice-gas automata for the Navier-Stokes equation. Phys Rev Lett 1986, 56: 1505-8.

26. Meinhardt $\mathrm{H}$, Klinger $\mathrm{M}$ : A model for pattern formation on the shells of molluscs. $J$ Theor Biol 1987, 126: 63-89.

27. Vichniac GY: Simulating physics with cellular automata. Physica D 1984, 10: 96-116.

28. Sander LM: Fractal growth. Scientific Am 1987 (Jan), 256: 94-100.
29. Witten TA Jr, Sander LM: Diffusion limited aggregation, a kinetic critical phenomenon. Phys Rev Lett 1981, 47: 1400-3.

30. Dewdney AK: Computer recreations: random walks that lead to fractal crowds. Scientific Am 1988 (Dec), 259: $116-9$.

31. Mainster MA: The fractal properties of retinal vessels: embryological and clinical implications. Eye 1990, 4: $235-41$.

32. Sprott JC and Rowlands G: Chaos Demonstrations. New York, American Institute of Physics, 1990.

33. Meakin P: Diffusion-controlled cluster formation in 2-6 dimensional space. Phys Rev A 1983, 27: 1495-507.

34. Niemeyer L, Pietronero L, Wiesmann HJ: Fractal dimension of dielectric breakdown. Phys Rev Lett 1984, 52: 1033-6.

35. Maloy KJ, Boger F, Feder J, Jossang T: Dynamics of viscous-fingering fractals in porous media. Phys Rev A 1987, 36: 318-24.

36. Wolfram S: Undecidability and intractability in theoretical physics. Phys Rev Lett 1985, 54: 735-8.

37. Wolfram S: Origins of randomness in physical systems. Phys Rev Lett 1985, 55: 449-52.

38. Lewis R: Practical Digital Image Processing. Chichester (WS), Ellis Horwood, 1990.

39. Phillips RP, Spencer T, Ross PGB, Sharp PF, Forrester JV: Quantification of diabetic maculopathy by digital imaging of the fundus. Eye 1991, 5: 130-7.

40. Ward NP, Tomlinson S, Taylor CJ: Image analysis of fundus photographs: the detection and measurement of exudates associated with diabetic retinopathy. Ophthalmology 1989, 96: $80-6$.

41. Goldberg RE, Varma R, Spaeth GL, Magargal LE, Callen D: Quantification of progressive diabetic nonperfusion. Ophthalmic Surg 1989, 20: 42-5.

42. Jindra LF, van Saarloos PP, Eikelbloom RH, Cooper RL: Fourier analyis of the nerve fibre layer by digital imaging techniques. Ophthalmic Surg 1989, 20: 370-2.

43. Webb RH, Hughes GW, Pomerantzeff O: Flying spot TV ophthalmoscope. Appl Optics 1980, 19: 2991-7.

44. Mainster MA, Timberlake GT, Webb RH, Hughes GW: Scanning laser ophthalmoscopy-clinical applications. Ophthalmology 1982, 89: 852-7.

45. Wolf S, Arend O, Toonen H, Bertram B, Jung F, Reim M: Retinal capillary blood flow measurement with a scanning laser ophthalmoscope-preliminary results. Ophthalmology 1991, 98: 996-1000. 